\title{
"The Department of Health and Human Services Action Plan to Reduce Racial and Ethnic Health Disparities: A Commentary on Data Needs to Monitor Progress Toward Health Equity"
}

\begin{abstract}
The 2011 Department of Health and Human Services (HHS) Action Plan to Reduce Racial and Ethnic Health Disparities (the Action Plan) signified an unprecedented federal commitment to eliminating racial and ethnic disparities in health and health care. HHS monitored the Action Plan so that stakeholders could understand whether it had been carried out as expected and was having its intended impact on disparities. This commentary (1) describes the key facilitators of HHS's process to monitor Action Plan implementation and (2) presents strategies to support data needs of others-such as HHS agencies and state and local governmentswho monitor and evaluate similar strategic plans to increase health equity. The strategies include: (1) incorporating monitoring from the outset of strategic planning, (2) defining the purposes of monitoring, (3) including outcome targets, (4) developing logic models and identifying indicators, (5) assessing available data sources, (6) building capacity for data collection and reporting, and (7) collecting and reporting data on race and ethnicity.
\end{abstract}

Keywords: Health disparities, Health services, health equity, Racial and Ethnic Health Disparities

\author{
Rashida Dorsey ${ }^{1}$, \\ Dana M. Petersen ${ }^{2}$ and \\ Lisa Schottenfeld ${ }^{2}$ \\ 1 Director, Division of Policy and Data, U.S. \\ Department of Health and Human \\ Services, Washington, DC, USA \\ 2 Mathematica Policy Research
}

Corresponding author: Rashida Dorsey Rashida.Dorsey@hhs.gov

Director, Division of Data Policy Office of the Assistant Secretary for Planning and Evaluation, U.S. Department of Health and Human Services, Washington, DC, USA.

Citation: Dorsey R, Petersen D, Schottenfeld L. "The U.S. Department of Health and Human Services Action Plan to Reduce Racial and Ethnic Health Disparities: A Commentary on Data Needs to Monitor Progress Towards Health Equity" Health Syst Policy Res. 2016, 3:4.

\section{Vision of the Health and Human Services Action Plan to Reduce Racial and Ethnic Health Disparities and its Emphasis on Monitoring}

Despite medical advances and new technologies that have improved Americans' quality of life and health status, longstanding health disparities exist between different racial and ethnic populations. All people should be able to live the healthiest lives possible and opportunities for good health should not be unevenly distributed according to race, ethnicity, or other characteristics. Disparities in health and health care are both unjust and persistent, so focused attention is needed to reduce and ultimately eliminate them. The vision of the 2011 Department of Health and Human Services (HHS) Action Plan to Reduce Racial and Ethnic Health Disparities (action plan) was to achieve "a nation free of disparities in health and health care.
The action plan signified an unprecedented federal commitment to eliminating racial and ethnic disparities in health and health care, and it is the first federal strategic plan to address this goal. It provided direction for other federal agencies, offered guidance for collaboration with communities and non-federal partners, and leveraged opportunities in the Affordable Care Act to benefit minority populations and reduce disparities. The action plan includes four secretarial priorities and five goals that sought to coordinate and integrate HHS minority health infrastructure and programs, and specified strategies intended to help HHS reduce disparities in health and health care. Table 1 shows examples of progress implementing these strategies. HHS reported results of monitoring efforts via the Reports to Congress on Minority Health Activities in 2013 and 2105 (organized around the five goals of the action plan) and the HHS action plan to Reduce Racial and Ethnic Health Disparities Implementation Progress Report in 2015. Agencies also regularly reported progress on implementing the Action Plan activities on their websites and shared information with ASPE and OMH. 
Table 1 Secretarial priorities, goals, strategies, and highlights of the HHS action plan to reduce racial and ethnic health disparities.

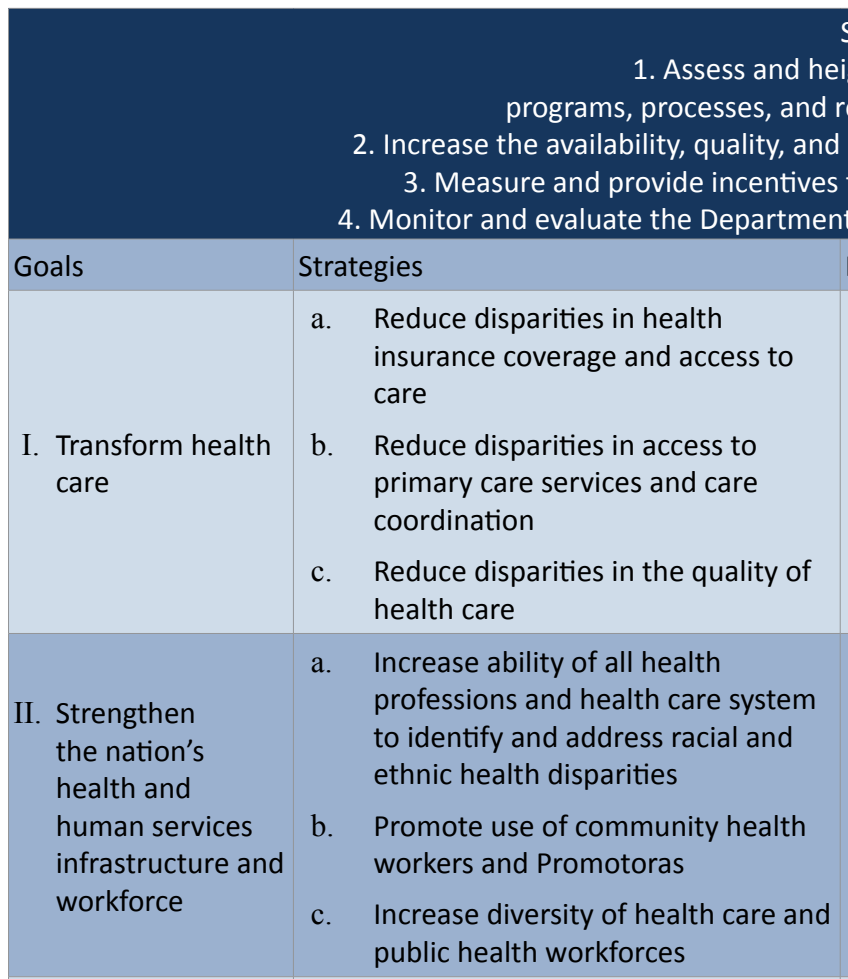

a. Reduce disparities in population health by increasing availability and

III. Advance the health, safety, and well-being of the American people

b. Conduct and evaluate pilot tests of health disparity impact assessments of selected national policies and programs

a. Implement a multifaceted health disparities data collection strategy across HHS

IV. Advance scientific knowledge and innovation

b. Conduct and support research to inform disparities-reduction initiatives

\section{Secretarial Priorities}

ighten the impact of all HHS policies,

resource decisions to reduce health disparities.

use of data to improve the health of minority populations.

or better health care quality for minority populations.

Implementation Progress Highlights

Under the Affordable Care Act (ACA), millions of racial and ethnic minorities gained access to affordable and quality health insurance coverage. HHS agencies took steps to improve access to coordinated primary care by establishing new community health centers in medically underserved communities, and assisting health centers to function as medical homes. Additionally, large-scale initiatives tested innovative models to improve the quality of cardiovascular disease prevention and care and dental services-two areas traditionally exhibiting racial and ethnic disparities in access to care and outcomes.

HHS agencies supported access to translation and interpretation assistance for individuals and provided information for health care providers about how to offer culturally competent care through the enhanced National Standards for Culturally and Linguistically Appropriate Services in Health Care. A national training curriculum was developed to support community health workers and Promotoras de Salud, along with recommendations for integrating these lay workers in health care settings. To help increase diversity in the health care workforce, the National Health Service Corps provided scholarships and loan repayment for racial and ethnic minority health care professionals.

Activities related to this goal including funding and assistance to numerous communities to design and implement local programs to prevent chronic illnesses such as cancer, diabetes, and heart disease. Strategies included establishing smoke- and tobacco-free policies, increasing the availability of healthy food and beverage options for children and families, home visiting programs, and influenza vaccination campaigns. Several agencies began requiring grantees to document the strategies they will use to promote health equity and to measure their impact on health-disparities reduction.

The goal fostered the promotion of new disparities focused research, including Patient-Centered Outcomes Research and groundbreaking longitudinal studies in tobacco. It also funded training for researchers interested in using community-based participatory research methods in projects that address health equity. HHS's adoption of new data standards (in accordance with section 4302 of the ACA) for the collection of race, ethnicity, sex, primary language, and disability status for self-reported data collected from national population-based surveys is an important advance.

HHS agencies are tracking, evaluating, and reporting on efforts to address health disparities so that results are optimized. The Action Plan itself identified 14 key population-based disparity measures that can be used for long-term monitoring of areas expected to be impacted by the Plan's investments.
V. Increase the efficiency, transparency, and accountability of HHS programs a. Streamline grant administration for health disparities funding

b. Monitor and evaluate implementation of the HHS Action Plan at the goal, strategy, and action levels.
The Action Plan's Secretarial Priority 4 ("Monitor and evaluate the Department's success in implementing the HHS Disparities Action Plan") and Goal V ("Increase the efficiency, transparency, and accountability of HHS programs") laid out the Department's approach to monitoring and evaluating the action plan's implementation and outcomes. Monitoring and evaluation efforts help policymakers and those developing and implementing a plan understand whether it has been carried out as expected and is having its intended impact. Monitoring can also help identify successful approaches that others can replicate, as well as any need for mid-course corrections to a plan. Data are a critical requirement for monitoring, as data provide the information necessary to systematically track implementation and outcomes; data are essential to help strategic plan developers and implementers identify the most valuable and efficient use of resources and make informed decisions about current operations and future directions in addressing health disparities. Since the release of the action plan, HHS actively used data to monitor its implementation.

This commentary describes the key facilitators of HHS's process to monitor action plan implementation. It also presents strategies and guidance to support data needs for monitoring based on 'HHS' experiences with the action plan that may be useful to others 
seeking to ensure appropriate data will be available to monitor and assess the implementation and outcomes of similar strategic plans- including HHS agencies and state and local governments.

\section{Key monitoring facilitators}

Two key factors facilitated monitoring of the action plan: (1) $\mathrm{HHS}^{\prime}$ commitment at multiple levels and (2) a monitoring infrastructure. High-level support from the Secretary of HHS signaled the Department's commitment to implementing and monitoring disparities reduction activities, fostering an understanding that collecting data and reporting progress on the action plan was a priority. Additional commitment from agency leadership, and the dedication of agency staff to track and report on disparities reduction efforts, helped to make data available to assess action plan progress and outcomes. The commitment of these internal champions, who were personally motivated to track disparities-reduction efforts, facilitated the reporting of data for monitoring purposes. Champions often included staff with a commitment to improving minority health and reducing health and health care inequities. Meaningfully involving and supporting these champions in monitoring efforts facilitated the collection of high quality data.

Infrastructure to support disparities reduction activities within HHS also facilitated efforts to monitor action plan implementation. The action plan tasked the Office of the Assistant Secretary for Health/Office of Minority Health (OASH/OMH) and the Office of the Assistant Secretary for Planning and Evaluation (ASPE) with leading the efforts to monitor implementation and report on progress. The Assistant secretaries of these offices co-chaired the HHS Health Disparities Council, which is composed of senior representatives from operating and staff divisions across HHS including the Deputy Assistant Secretary for Minority Health, the directors of the agency-level Offices of Minority Health, and the director of the National Institute on Minority Health and Health Disparities. The Council oversaw action plan implementation and monitoring, and a subgroup of the Council provided guidance on the design and implementation of monitoring activities. $\mathrm{OMH}$ served as executive director of the Council and worked with members to ensure that data collection efforts would support an accurate assessment of action plan activities and outcomes. Likewise, Council members provided leadership and support within their respective agencies to coordinate data collection and reporting. Furthermore, staff with substantive and methodological expertise in monitoring disparities reduction, evaluation, and data provided support to help agencies report progress on activities in the action plan.

\section{Strategies for successful monitoring}

The inclusion of Secretarial Priority 4 and Goal V in the action plan signaled HHS's commitment to investing the resources required to monitor implementation and progress toward goals at the outset of the action plan. In this section, we present selected strategies to support data needs for monitoring for others seeking to track implementation of strategic plans to promote health equity. These strategies come from the authors' experiences monitoring action plan implementation, including agency data on the implementation and outcomes of activities in the action plan, and conversations with HHS staff directly involved with data collection and reporting. Most of the guidance relates to decisions that should occur while developing a strategic plan, which should involve discussions about plan monitoring and implementation.

\section{Define the purposes for monitoring}

Within the context of any strategic plan, the purposes for monitoring dictate the types of data required to evaluate outcomes accurately. Conversely, data can also help shape the purpose of the strategic plan. For instance, if data are not available for a proposed purpose, that purpose may need to be revised so that strategies and action steps that can be appropriately monitored using data. Given that HHS' primary purposes for monitoring the five-year plan were to track implementation and short-term outcomes, agencies regularly shared descriptive information on activities conducted and short-term results. Longer-term monitoring of changes in population-level disparities in health and health care would require longitudinal data on indicators related to disparities reduction activities among the populations served. Although HHS did not engage in longer-term monitoring because of the short-term nature of the action plan, the department did identify certain measures collected in major HHS surveys that could be monitored in the future or retrospectively.

\section{Develop logic models and identify indicators}

Shortly after HHS released the action plan, it developed a logic model illustrating the hypothesized relationships among action plan goals, outputs, and outcomes (Figure 1). HHS also developed more specific logic models for each goal that depicted the strategies and activities likely to achieve them. As a set, the logic models showed how the strategies and activities in the action plan would work together to bring about desired reductions in disparities. HHS used these models to refine and develop process, short-term, intermediate, and long-term outcome indicators that could be used to test the hypotheses depicted in the models and monitor the implementation and outcomes of the action plan. Developing logic models is critical to establishing effective monitoring processes. Indicators included in logic models inform strategic plan data needs, and provide a roadmap to support the collection of appropriate data for monitoring purposes. They help to foster a shared understanding of a plan by uncovering assumptions about how the activities and strategies in the plan relate to one another and exploring implicit hypotheses about how activities and strategies contribute to disparities reduction outcomes and goals. Logic models also help set priorities for data and monitoring by identifying the activities and strategies most central to the plan's overall success, and associated indicators. 


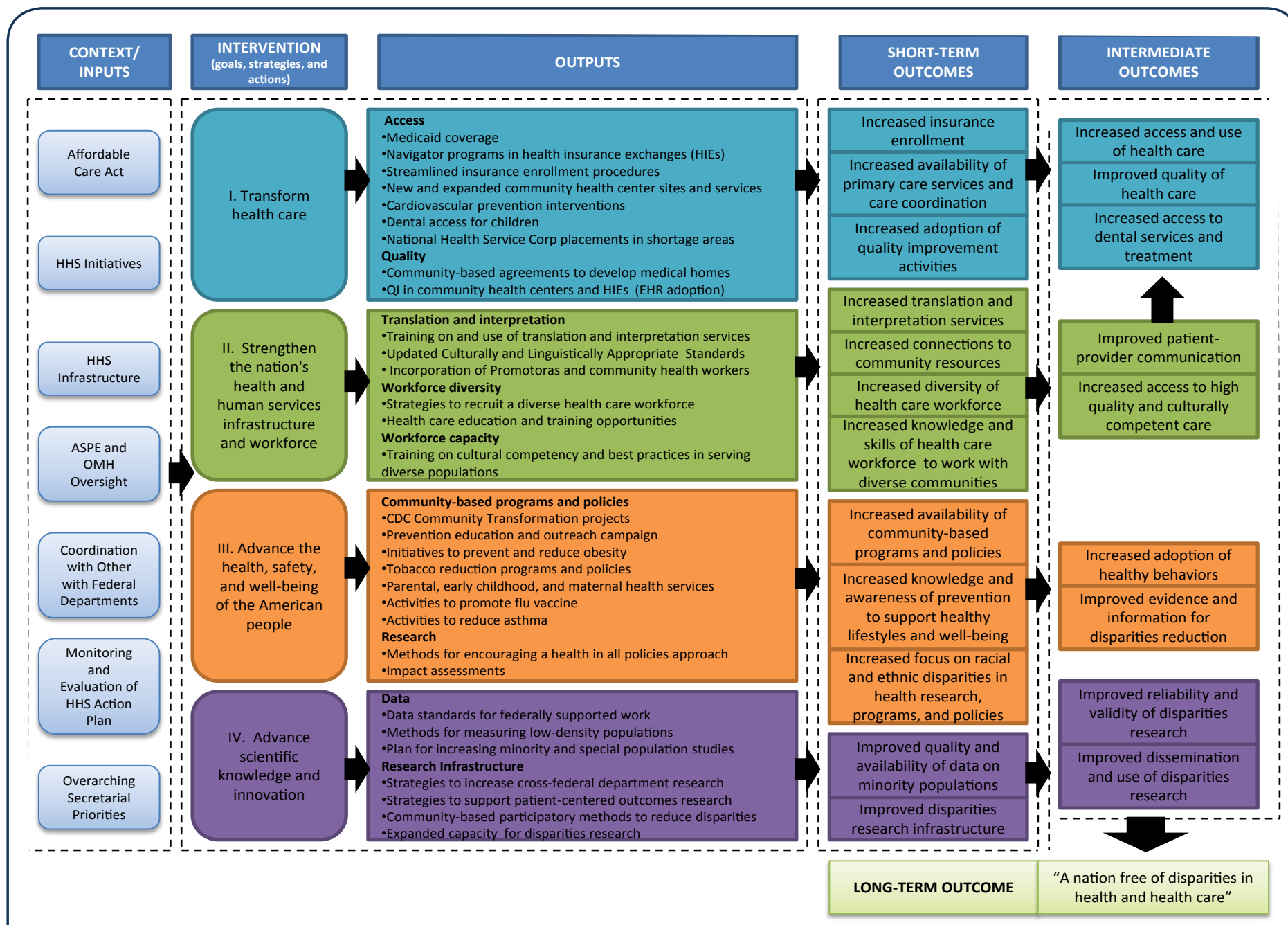

Figure 1 Action plans, goals, outputs, and outcomes.

\section{Assess data sources available for monitoring}

The robustness of monitoring efforts is dependent upon the data available. Therefore, understanding the data that can be obtained through existing resources, including strengths and limitations, as well as access and use of datasets, is essential for monitoring. Throughout the implementation of the action plan, HHS reviewed data available to monitor progress. Most agencies could easily report qualitative data, but some agencies faced challenges in specifying and collecting quantitative outcome measures to track over time. Tension often arises between a desire to rely only on existing data sources and the need to create new data sources to measure progress toward goals. This can be particularly challenging if the existing data primarily measure processes rather than outcomes. Strategic plan developers and implementers should be involved in decisions about when and how to invest in developing new data sources or refine existing ones to track meaningful progress toward strategic plan goals. We suggest that strategic plan developers work with stakeholders involved with implementation and data collection to identify feasible data sources. Implementers can provide key information about the types of data collection that will be possible given resource constraints.

\section{Leverage capacity for data collection and reporting}

Because strategic plan implementers may face challenges in collecting and reporting data, we recommend that those monitoring disparities-related strategic plans work with stakeholders to identify and prepare for anticipated challenges. Intentional collaborations between stakeholders such as programmatic staff, performance officers, and data experts can leverage capacity for data collection and monitoring. Helping implementers to assess their own capacity, or leverage the capacity of others, to identify and collect meaningful, feasible indicators that they can report at regular intervals can result in higher quality qualitative and quantitative longitudinal data. Technical assistance on data collection throughout a plan's implementation can improve monitoring and evaluation. 


\section{Collect and report data on race and ethnicity}

For strategic plans that seek to address health equity, it is important to track progress among key racial and ethnic subgroups, rather than solely reviewing progress in the full population. To monitor long-term progress of a plan that seeks to eliminate disparities among racial and ethnic populations, process and outcome measures must adhere to uniform standards for collecting demographic data and be disaggregated by race, ethnicity, and other characteristics of interest. If the categories used to collect race and ethnicity data are not standardized, it is difficult to aggregate results across plan activities and monitor their overall influence on disparities. Staff training on the established standards for collecting and reporting race and ethnicity data may also be important. The data collection standards for race and ethnicity promulgated by the Office of Management and Budget in 1997 supported HHS's monitoring efforts. In addition, HHS adopted more detailed race and ethnicity standards in 2011 for its major population-based surveys.

\section{Include targets and milestones}

Setting targets defines and establishes standards for success, communicates expectations for meeting outcomes, and helps to place outcomes in context (for example, by indicating whether they fell below, met, or exceeded expectations). Although most agencies established their own milestones for disparities reduction activities included in the action plan, and although the action plan did include goal statements, it did not directly link its activities or strategies to specific outcome targets. Defining achievable short-term targets is critical to assessing a plan's success, particularly when the longer-term goals address longstanding, complex issues such as health and health care disparities. Including experts in strategic plan evaluation can help plan developers describe clear, measurable goals and set targets to evaluate progress.

\section{Conclusion}

The establishment of the action plan signified a major advance in federal efforts to eliminate racial and ethnic disparities in health and health care. However, creating a strategic plan is not enough to ensure implementation and effective performance. Efforts to monitor and evaluate the action plan demonstrated HHS' commitment to work toward its goal of a nation free of disparities in health and health care and the Department's ongoing investment in developing and improving data collection and reporting to track progress toward equity. The data collected, reported and analyzed as part of monitoring efforts supported HHS efforts to identify the most valuable and efficient use of resources and make informed decisions about current operations and future directions in addressing health disparities.

Establishing a monitoring system enabled HHS to track movement toward the action plan's goals and promote optimal performance. Robust monitoring structures such as these can identify the achievement of strategic plan goals as well as any need for refinements or changes in direction. Monitoring can also help organizations implementing strategic plans to identify strategies for successful replication, opportunities to improve the timeliness and quality of results, and methods for reducing duplication or aligning and leveraging resources wisely. The department's experiences in creating this monitoring system can inform efforts to evaluate other strategic plans to address health equity. This could include future efforts by HHS agencies or by other state or national health agencies. In each of these types of settings, staff can help create more effective systems to monitor progress toward health equity by applying the strategies described here to [1] include monitoring from the outset of strategic planning, [2] identify the purposes of monitoring, [3] include outcome targets, [4] develop logic models and identify relevant indicators, [5] assess available data sources and create new ones if needed, [6] build capacity for data collection and reporting, and [7] collect and report data on race and ethnicity.

\section{Disclaimer}

The findings in this report represent those of the authors and do not necessarily represent those of the Department of Health and Human Services or the Office of the Assistant Secretary for Planning and Evaluation. 


\section{References}

1 Moy E, Freeman W (2014) Federal investments to eliminate racial/ ethnic health-care disparities. Public Health Reports 129: 62-70.

2 (2011) HHS Action Plan to Reduce Racial and Ethnic Health Disparities:A Nation Free of Disparities in Health and Health Care. U.S. Department of Health and Human Services, Washington, DC.

3 Koh HK, Graham G, Glied SA (2011) Reducing racial and ethnic disparities: the action plan from the Department of Health and Human Services. Health Affairs 30: 1822-1829.

4 (2013) Health Report to Congress on Minority Health Activities as Required by the Patient Protection and Affordable Care Act:For the
Years 2011 and 2012. U.S Dept. of Health and Human Services, Office of the Secretary, and Office of Minority Health Washington, DC.

5 (2015) Report to Congress on Minority Health Activities as Required by the Patient Protection and Affordable Care Act: For the Years 2013 and 2014. U.S. Dept. of Health and Human Services, Office of the Secretary, and Office of Minority Health. Washington, DC.

6 (2015) HHS Action Plan to Reduce Racial and Ethnic Health Disparities Implementation Progress Report. U.S. Dept. of Health and Human Services, Office of the Secretary, Office of the Assistant Secretary for Planning and Evaluation and Office of Minority Health.. Washington, DC.

7 https://aspe.hhs.gov/basic-report/hhs-action-plan-reduceracial-and-ethnic-health-disparities-implementation-progressreport-2011-2014. 